\title{
Simple, but not so simple
}

In science, some of the trickiest things to explain - especially for non-scientists involve ideas that seem obviously true, but which might actually be true only for very non-obvious reasons. I encountered the problem several years ago when writing a popular article about unorthodox interpretations of quantum mechanics that allow the assignment of precise trajectories to quantum particles. Making this at all interesting meant first explaining why physicists had given up on this deeply intuitive and seemingly obvious idea long ago, and only then explaining why further work in fact showed it to be possible after all, at least in principle. Without a lot of preliminary discussion, people would simply think: so what?

Now I realize that I've been in the same confused position in thinking about evolution. I thought I understood, but actually I didn't. Yet now that I do understand, it seems I can go on thinking pretty much what I thought before. (Or maybe I'm confused on a higher level, as Enrico Fermi once said.)

In my mental model of the evolution of a population of organisms, a swarm of dots the organisms - move about in some abstract and high-dimensional fitness space. Of course, we know that evolutionary search is driven by mutations and natural selection. I imagine the swarm moving about and through chance mutations gradually discovering better 'designs'. It generally becomes fitter over time, until reaching some local niche from which further improvement through small mutations is extremely unlikely.

At this point, in my mind, there is a balanced equilibrium supported by countervailing processes. Natural selection on its own should tend to focus the swarm more closely about the fittest genotype. Yet mutations - most of which decrease fitness - should counter this narrowing and keep the population spread out in the fitness space. This is a simple enough picture, but is in, its details, definitely wrong. Fortunately, it seems that I only have to revise it a little, as Sidhartha Goyal and colleagues have demonstrated with considerable calculation backed by computer simulations (http://arXiv.org/abs/1110.2939; 2011).

The problem with my 'obvious' picture was pointed out long ago by biologist Hermann Joseph Muller, and is known as Muller's ratchet. It applies to any finite

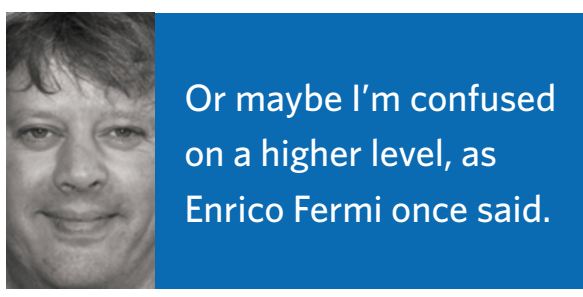

population, but to smaller ones more strongly. Suppose we divide the population into groups - a fittest group, second fittest group and so on. Muller noted that there is always a chance in any generation that the fittest group will have no offspring. Given time, this has to happen. The fittest group will then disappear.

The second fittest now becomes the fittest. But then the same thing will happen to the second fittest group, and the third, and so on. These groups should progressively disappear; the population should grow less fit by random chance alone. Eventually, this process can lead to so-called mutational meltdown, in which a population (especially a small one) accumulates mutations, loses average fitness, grows smaller and therefore more susceptible to the ratchet, and may ultimately go extinct.

Natural selection alone - as I had supposed in my intuitive picture - just isn't enough to do the job of keeping the population in equilibrium. But the world is, of course, full of finite populations. Why? Something must stop the ratchet. What is it?

In principle, it could be so-called back mutations - exceptional genetic errors that increase rather than decrease fitness - but for a well-adapted population, biologists have long supposed, these would be too rare to be of importance. After all, for a population near a fitness optimum, any random change is almost certainly going to be bad. Another possibility is sexual recombination, which allows the combination off two only slightly mutated genomes to create next-generation organisms without any deleterious mutations. Indeed, biologists have proposed Muller's ratchet as the primary reason that sex exists, as it counters the effect of the ratchet very effectively. It has also been taken to explain the lack of many asexual populations.

So Muller's logic completely destroys my intuitive picture in which natural selection alone is enough to counter the degrading forces of mutation. But Goyal and colleagues have now shown that the picture can hang together if natural selection is aided by beneficial mutations, even if these occur very rarely. The simple back-mutation idea turns out to be sensible after all.

Their basic idea is that the action of the ratchet itself, after a time, should begin to make it more likely for some mutations to be beneficial rather than harmful. For a highly adapted population, the fraction of all mutations that are beneficial, call it $\varepsilon$, will indeed be very small. But as the ratchet works and the population becomes less adapted, it should become more likely for some mutations to be beneficial. With falling fitness, moreover, $\varepsilon$ should continue to grow until these beneficial mutations become just strong enough to stabilize the population. If the fitness of the population fell below this level, beneficial mutations would be even more likely and should drive the fitness back up. There should, then, be a critical value of $\varepsilon$ where the effects of harmful and beneficial mutations just balance. "We expect", as they put it, "the dynamic mutation-selection balance point to be a stable evolutionary 'attractor.'”

The authors go on to show how the critical equilibrium value of $\varepsilon$ depends on several key factors such as population size, overall mutation rate and strength of selection, which would be determined by the environment. The key result is that the balance can happen at a point where the population has a high fitness - and should therefore be able to persist. At this balance point, most mutations by far are deleterious, and only very few are beneficial - as is true in most real populations.

Strikingly, this turns out to be true even for small populations, where beneficial mutations are much more effective in countering Muller's ratchet than previously thought. Goyal et al. go on to explore several real populations of asexual organisms viruses and the bacterium Escherichia coli in laboratory experiments - which seem to fit this scenario quite closely. In the case of E. coli, the critical value of $\varepsilon$ can be as low as $10^{-10}$.

In the end, this is a delightfully simple story, though the work needed to demonstrate its coherence isn't so simple. It means I can retain my simple intuitive picture of how populations maintain themselves, just by adding a few rare beneficial mutations to the work of natural selection. I was right all along, I just didn't deserve to be.

MARK BUCHANAN 